\title{
Control Loop Pairing and Interaction Analyses of the Falling Film Evaporator Process
}

\author{
Julian Hofmann ${ }^{\star}$, Lutz Gröll ${ }^{\dagger}$, Veit Hagenmeyer ${ }^{\ddagger}$ \\ Institute for Automation and Applied Informatics \\ Karlsruhe Institute of Technology \\ Eggenstein-Leopoldshafen, Germany, \\ `julian.hofmann@kit.edu, †lutz.groell@kit.edu, ${ }^{\ddagger}$ veit.hagenmeyer@kit.edu
}

\begin{abstract}
The paper solves the control loop pairing problem of the falling evaporator process. To this end, we derive a dynamic input-output model by taking dominant time-delays into account. Besides classical methods to solve the loop pairing problem, such as Bristol's relative gain array or the Niederlinski index, we apply modern approaches, namely, the participation matrix and Hankel interaction index array. Since the former only focus on multiloop single-input-single-output control, the latter additionally give recommendations regarding the structure of a multivariable controller. On the one hand, we show a feasible pairing for multiloop single-input-single-output process control but, on the other hand, we conclude a triangular structure of a multivariable controller, which may achieve better results due to the strong interaction of two control loops.
\end{abstract}

Index Terms-Control Loop Pairing, Falling Film Evaporator, Relative Gain Array, Gramian-Based Interaction Measures

\section{INTRODUCTION}

Numerous process engineering applications are designed as multiple-inputs-multiple-outputs (MIMO) plants. After classifying the inputs into manipulated variables and disturbances, the pairing problem of controlled and manipulated variables naturally arises. Practically, this problem is often solved intuitively by applying a decentralized multiloop single-inputsingle-output (SISO) control architecture, which however can lead to poor performance [1]. Nevertheless, a deep analysis of the pairing problem and investigating possible loop interactions help to find a more efficient multivariable control structure [2], [3]. To this end, the following questions should be answered:

1) Which pairing choice of controlled and manipulated variables is best for multiloop SISO control?

2) Is this pairing choice stable over the relevant plant's operation frequency domain?

3) Is this pairing choice feasible regarding stability of the multiloop SISO control system?

4) Is it better to design a multivariable controller instead of multiloop SISO controllers?

5) If yes, how should we structure this multivariable controller?

By inventing the famous relative gain array (RGA), Bristol

[4] presented a steady-state measure giving an answer to

The authors thank GEA Wiegand GmbH for financial support and for providing data used in this paper.
Question 1). Subsequently, many extensions of the RGA were created, cf. [5]-[7]. In this context, substituting the steadystate gain matrix by the transfer function matrix in Bristol's RGA is most common [2] and solves Question 2). The latter approach is referenced as dynamic relative gain array (DRGA) throughout the present paper. Although the steady-state RGA and DRGA are often applied to various processes [8]-[10], Question 3) cannot be answered by these methods. To remedy this issue, there exist the notions of decentralized integral controllability (DIC) [2], [11] and the Niederlinski index (NI) [12]. Since all of the aforementioned process measures assume multiloop SISO control, it is debatable whether a multivariable controller achieves better performance, see Question 4). Therefore, modern approaches, such as the participation matrix (PM) [13] or the Hankel interaction index array (HIIA) [14], use the Gramian controllability and observability matrices to quantify process interactions. Consequently, recommendations on the structure of a multivariable controller are obtained, which answers Question 5).

In this paper, we solve Questions 1)-5) for the falling film evaporator (FFE) process by applying RGA, DRGA, NI, DIC, PM and HIIA. The work coming closest to ours is [15], where RGA and DRGA are used to decide which manipulated variable is best to control the output dry matter content of the FFE process. Based on a different model than the one presented in [15], we verify the results obtained therein and extend them by investigating the feasibility of a chosen pairing for multiloop SISO control. Furthermore, we analyze if a multivariable controller may achieve better results than multiloop SISO controllers. Finally, we give recommendations w.r.t. a possible structure of the multivariable controller.

The plan of the paper is as follows. In Sec. II, we recapitulate all needed methods and explain the FFE process, whose model is introduced in Sec. III. Our main contribution is presented in Sec. IV, where we apply the methods of Sec. II to the model of Sec. III. In Sec. V, we sum up our results and give an outlook on future work.

\section{Methods And Materials}

In this section, we present the most important definitions, properties, theorems, criteria and recommended actions for all considered methods. For more detailed information, the 
reader is referred to the given references. Moreover, we briefly introduce our practical application, the FFE process.

\section{A. Relative Gain Array}

The relative gain array (RGA) of a nonsingular square transfer function matrix $G(s)$ being stable is defined by

$$
R(s)=G(s) \circ\left(G(s)^{-1}\right)^{\top},
$$

where $\circ$ is the Hadamard product. Firstly, note that generalizations to unstable $G(s)$ are known [16]. Secondly, note that the steady-state RGA, see Sec. II-A1, and the DRGA, see Sec. II-A2, can be directly applied to $G(s)$ with timedelays [17]. Additionally, DRGA enables conclusions on the existence of right half-plane (RHP) transmission zeros in $G(s)$ or RHP-zeros in its elements $g_{i j}(s)$ [18]. Thirdly, note that the RGA is independent of the scaling of $G(s)$. Fourthly, note that the RGA is normalized such that the sum of each column, as well as the sum of each row, is equal to one. The essential recommendation is to prefer pairings with RGAelements close to one and to avoid parings with negative RGAelements.

1) Steady-State: If (1) is evaluated at $s=0$, we obtain the steady-state RGA $R(0)$, cf. [2], [4].

2) Dynamic: If (1) is evaluated at $s=\mathrm{i} \omega$, where i denotes the imaginary unit and $\omega$ the circular frequency, we obtain the DRGA $R(\mathrm{i} \omega)$, cf. [2], [19]. Some advice on determining the relevant frequency domain of a plant is given in Sec. IV and in [8]. To practically evaluate $R(\mathrm{i} \omega)$, it is common to consider the magnitude of its elements $\left|r_{i j}(\mathrm{i} \omega)\right|$ over $\omega$. In this case, the aforementioned normalization property of the RGA is not valid anymore. Furthermore, negative RGA-elements are no more possible, which may lead to misinterpretations. However, these drawbacks can be mitigated by comparing $|R(\mathrm{i} \omega)|$ to the steady-state RGA, see also Sec. IV.

\section{B. Decentralizability Quantities}

While Niederlinski's theorem [12], [20] provides a sufficient condition for instability of the multiloop SISO control system, the notion of decentralized integral controllability makes a statement on the existence of a stabilizing diagonal controller with integral action. The relation between both concepts is precised in [21]. Additionally, note that both of the decentralizability quantities, the Niederlinski index and decentralized integral controllability, depend on the chosen pairing but are independent of the scaling of $G(s)$. To avoid recalculating these quantities for each possible paring, we recommend to apply the steady-state RGA and DRGA in advance and only evaluate the obtained loop pairing recommendations.

1) Niederlinski Index: The following theorem was originally introduced in [12].

Theorem 1 (Niederlinski's theorem, refined version [20]). Consider a closed-loop system composed of the square plant $G(s)$ and the diagonal controller

$$
K(s)=\frac{k}{s} \underbrace{\operatorname{diag}\left(\tilde{k}_{1}(s), \tilde{k}_{2}(s), \ldots, \tilde{k}_{m}(s)\right)}_{=\tilde{K}(s)}, \quad k>0,
$$

where $m$ is the number of plant inputs being equal to the number of plant outputs and $\tilde{k}_{j}(s), j=1,2, \ldots, m$ denotes controllers without integrators. Let us assume that the following conditions hold:

(a) $G(s)$ is stable,

(b) $H(s)=G(s) \tilde{K}(s)$ is rational and proper,

(c) each individual control loop is stable if any of the other loops is opened.

Then, a sufficient condition for instability of the closed-loop system is

$$
\underbrace{\frac{\operatorname{det} G(0)}{\prod_{j=1}^{m} g_{j j}(0)}}_{=\mathrm{NI}}<0 .
$$

Thus, Theorem 1 excludes unfeasible pairings in multiloop SISO control systems. Firstly, note that the left-hand side of (3) is called Niederlinski index (NI). Secondly, under the assumptions of Theorem 1, NI $>0$ is a necessary condition for stability of the multiloop SISO control system [20]. Thirdly, note that the diagonal proper controller $K(s)$ in (2) represents all controllers of interating type, e.g., I, PI or PID [12]. Hence, a cancellation of the zero-pole in $K(s)$ is not allowed. Fourthly, note that Assumption (c) of Theorem 1 is also referenced as integrity, cf. [22].

2) Decentralized Integral Controllability: The definition is given as follows.

Definition 1 (Decentralized Integral Controllability [11]). The square plant $G(s)$ is decentralized integral controllable (DIC) if there exists a diagonal controller $K(s)$ with integral action in each loop such that the closed-loop system is stable and such that each individual loop may be detuned independently by a factor $\varepsilon_{j}, 0 \leq \varepsilon_{j} \leq 1, j=1,2, \ldots, m$ without introducing instability.

The following criteria are, e.g., given in [21] and hold for stable $G(s)$. While a necessary condition for DIC

$$
r_{j j}(0) \geq 0, \quad j=1,2, \ldots, m
$$

uses the diagonal elements $r_{j j}(0)$ of $R(0)$, a sufficient one

$$
\mu(E(0))<1
$$

is based on the structured singular value $\mu$, see [23] for its definitions, of the matrix

$$
E(0)=\left(G(0)-G_{\operatorname{diag}}(0)\right) G_{\operatorname{diag}}(0)^{-1},
$$

where

$$
G_{\text {diag }}(0)=\operatorname{diag}\left(g_{11}(0), g_{22}(0), \ldots, g_{m m}(0)\right) .
$$

Firstly, note that $\mu(E(0))$ can be determined numerically in Matlab via mussv $(E$, ones $(\operatorname{size}(E, 1), 2))$ since $\mu$ is computed w.r.t. the structure of $K(s)$, see (2) and [21]. Secondly, note that there are also other criteria for $2 \times 2$ and $3 \times 3$ plants, cf. [2]. Thirdly, note that DIC can be related to the concept of passivity, which is detailed in [11]. 


\section{Gramian-based Interaction Measures}

At first, let us recall the definitions of the Gramian controllability matrix

$$
P=\int_{0}^{\infty} \mathrm{e}^{A \theta} B B^{\top} \mathrm{e}^{A^{\top} \theta} \mathrm{d} \theta
$$

and the Gramian observability matrix

$$
Q=\int_{0}^{\infty} \mathrm{e}^{A^{\top} \theta} C^{\top} C \mathrm{e}^{A \theta} \mathrm{d} \theta,
$$

where the matrices $A \in \mathbb{R}^{n \times n}, B \in \mathbb{R}^{n \times m}$ and $C \in \mathbb{R}^{m \times n}$ correspond to the minimal spate space representation of the stable plant $G(s) \in \mathbb{C}^{m \times m}$. Subsequently, we introduce two Gramian-based interaction measures called participation matrix and Hankel interaction index array. These Gramianbased measures represent matrices, depend on the scaling of $G(s)$ and yield the following recommendations [19]:

- The larger the element of the measure matrix, the larger the impact of the corresponding input on the corresponding output.

- The measure matrix elements sum, which is close to one, determines the control structure.

Since the Gramian-based measures are introduced for delayfree $G(s)$, it is convenient to approximate delay terms $\mathrm{e}^{-s \tau}$ via a Padé approximation [19]. Moreover, both Gramianbased measures are similarity invariants and therefore directly applicable to $G(s)$.

1) Participation Matrix: According to [13], the elements $\phi_{i j}$ of the participation matrix (PM) $\Phi$ are defined by

$$
\phi_{i j}=\frac{\operatorname{tr}\left(P_{j} Q_{i}\right)}{\operatorname{tr}(P Q)},
$$

where $P_{j}$ and $Q_{i}$ are the controllability Gramian and observability Gramian, respectively, for the subsystem $(i, j)$, i.e., $g_{i j}(s)$. Thus, the elements of $\Phi$ correspond to the input $u_{j}$ and to the output $y_{i}$. To be able to compare $\Phi$ to other Gramian-based measures, it is beneficial [19] to consider the normalization

$$
\bar{\phi}_{i j}=\frac{\sqrt{\phi_{i j}}}{\sum_{l=1}^{m} \sum_{k=1}^{m} \sqrt{\phi_{k l}}} .
$$

2) Hankel Interaction Index Array: Let us start by recapitulating the Hankel singular values (HSVs) of $G(s)$ with Gramians $P$ and $Q$. The HSVs are given by

$$
\sigma_{\mathrm{H}, i}=\sqrt{\lambda_{i}(P Q)}, \quad i=1,2, \ldots, n,
$$

where $\lambda_{i}((\cdot))$ denotes the eigenvalues of $(\cdot)$ and the Matlab command to calculate the HSVs of a system sys is hsvd (sys). Furthermore, the Hankel seminorm of $G(s)$ is determined by

$$
\|G(s)\|_{\mathrm{H}}=\max _{1 \leq i \leq n} \sigma_{\mathrm{H}, i} .
$$

Let $\left\|g_{i j}(s)\right\|_{\mathrm{H}}$ denote the Hankel seminorm of the $(i, j)$ subsystem of $G(s)$, then, according to [14], the $(i, j)$-th element of the normalized Hankel interaction index array (HIIA) $H$ is determined by

$$
h_{i j}=\frac{\left\|g_{i j}(s)\right\|_{\mathrm{H}}}{\sum_{l=1}^{m} \sum_{k=1}^{m}\left\|g_{k l}(s)\right\|_{\mathrm{H}}} .
$$

\section{Falling Film Evaporator Process}

The scheme of a falling film evaporator (FFE) with four passes is depicted in Fig. 1. Its main purpose is to increase the dry matter content of some liquid, e.g., milk, to get the desired output dry matter content $w_{o}$. Besides the latter, the output mass flow $\dot{m}_{o}$ is another variable to be controlled. Via Plate 1 , the input liquid with mass flow $\dot{m}_{i}$ and dry matter content $w_{i}$ is distributed over the Tubes of the first pass, where it flows down as thin film and falls into Reservoir 1. Some vapor is directed to the outside of the Tubes to initiate evaporation of the liquid on the inside. After the ramp-up via the live steam vapor mass flow $\dot{m}_{\mathrm{v} \text {,init }}$, the process is able to operate with its self-generated vapor. The latter is conveyed from the Compressor's suction side, called Effect, to the Compressor's pressure side, called Heat Chamber. Due to the Compressor's action, the additional power $P_{\mathrm{C}}$ is supplied to the process. To keep the energy in the Heat Chamber and thus in the Effect balanced, some vapor mass flow $\dot{m}_{\mathrm{v}, \text { con }}$ is taken out of the Heat Chamber. Hence, besides controlling $w_{o}$ and $\dot{m}_{o}$, the third aim consists in controlling the Effect's temperature $\vartheta_{\mathrm{E}}$. To enable large $w_{o}$, the process has a cascade-like structure, i.e., the liquid out of Reservoir 1 is pumped through Pipe 1 into the second pass starting with Plate 2, etc. Thus, the Pipes and Tubes modules affect dominant transport delays, which makes control of $w_{o}$ and $\dot{m}_{o}$ challenging. The nomenclature used in Fig. 1 and Sec. III is shown in TABLE I.

\section{Modeling}

The aim of this section is to derive a control-oriented model of the whole FFE process. To this end, we derive equations relating the controlled variables $w_{o}, \dot{m}_{o}$, and $\vartheta_{\mathrm{E}}$ to the manipulated variables $P_{\mathrm{C}}, \dot{m}_{i}$, and $\dot{m}_{\mathrm{v}, \text { con }}$. As origin, we consider the complex modular models presented in [24], [26]. However, since these models are too detailed for the intended pairing and interaction analyses, we instead focus on the rearranged flow diagram in Fig. 2 by making use of only important module dynamics. The advantage of rearranging the flow diagram as shown in Fig. 2 offers the opportunity to aggregate all transport delays of Pipes and Tubes elements. This notion will lead us to practical relations for $w_{o}$ and $\dot{m}_{o}$, see (26) and (27). Moreover, we evaluate energy balances of the Effect and Heat Chamber to get relations for $\vartheta_{\mathrm{E}}$ and $\vartheta_{\mathrm{H}}$. After describing all modules, we introduce control nomenclature and combine all model equations to a nonlinear ODE system with input delays. The latter is linearized about the operation point, which yields a transfer function matrix representation being compatible for further analyses. 


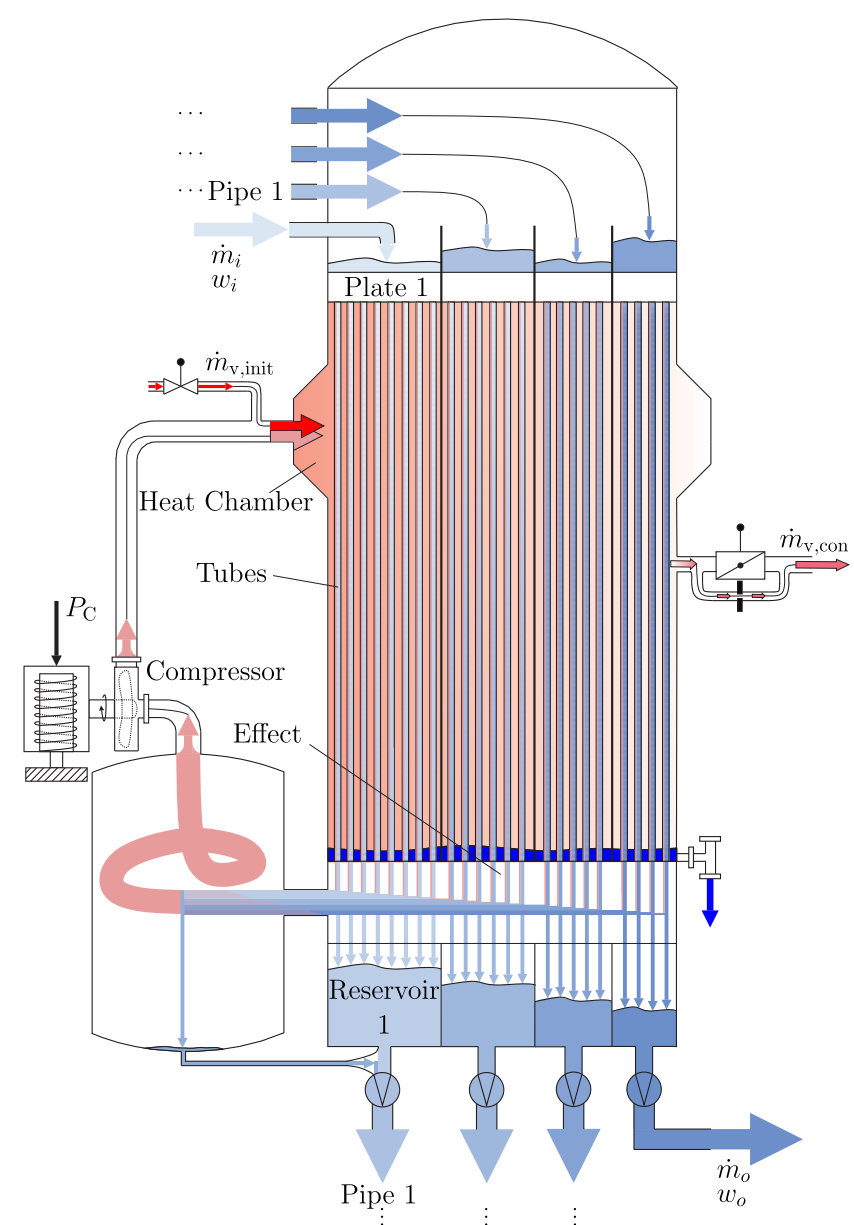

Fig. 1: Scheme of a falling film evaporator, cf. [24], [25]

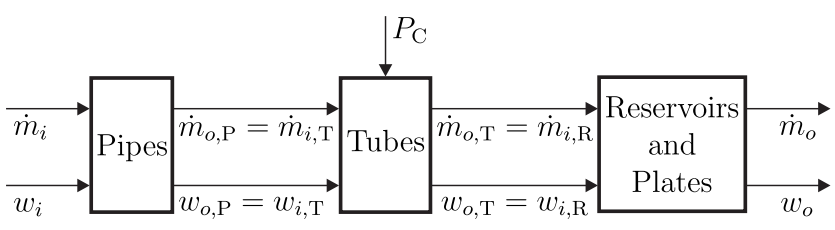

Fig. 2: Rearranged flow diagram of the FFE process

\section{A. Modules}

In the following, the dynamics of relevant FFE modules are specified.

1) Pipes: According to [24], [26], the transport in Pipes is modeled as feedthrough w.r.t. mass flow, i.e.,

$$
\dot{m}_{o, \mathrm{P}}(t)=\dot{m}_{i}(t)
$$

and can be approximated as constant delay of dry matter content, i.e.,

$$
w_{o, \mathrm{P}}(t)=w_{i}\left(t-\tau_{\mathrm{P}}\right) .
$$

2) Tubes: As described by [26], the input-output equations of FFE Tubes are modeled by

$$
\dot{m}_{o, \mathrm{~T}}(t)=\dot{m}_{i, \mathrm{~T}}\left(t-\tau_{\mathrm{T}}\right)-\frac{1}{\tau_{\mathrm{T}}} \int_{t-\tau_{\mathrm{T}}}^{t} \dot{m}_{\mathrm{v}}(\theta) \mathrm{d} \theta,
$$

TABLE I: Symbol and subscript nomenclature

\begin{tabular}{l|l|l}
\multicolumn{2}{l}{ Symbol } & Unit \\
\hline$A$ & cross-sectional area & $\mathrm{m}^{2}$ \\
$c_{p}$ & specific heat capacity & $\mathrm{J} \mathrm{kg}^{-1} \mathrm{~K}^{-1}$ \\
$\Delta h_{v}$ & enthalpy of evaporation & $\mathrm{J} \mathrm{kg}^{-1}$ \\
$k$ & heat transfer coefficient & $\mathrm{W} \mathrm{m}-2 \mathrm{~K}^{-1}$ \\
$\dot{m}$ & mass flow & $\mathrm{kg} \mathrm{h}^{-1}$ \\
$M$ & mass & $\mathrm{kg}$ \\
$N$ & rotational speed & $\mathrm{rpm}$ \\
$p$ & pressure & $\mathrm{kPa}$ \\
$P$ & power & $\mathrm{W}$ \\
$\dot{q}$ & heat flow & $\mathrm{W}$ \\
$t$ & time & $\mathrm{s}$ \\
$T$ & low pass filter time constant & $\mathrm{s}$ \\
$w$ & dry matter content & $\mathrm{kg} \mathrm{kg}$ \\
$\vartheta$ & temperature & ${ }^{\circ} \mathrm{C}$ \\
$\varrho$ & volumetric mass density & $\mathrm{kg} \mathrm{m}^{-3}$ \\
$\tau$ & time-delay & $\mathrm{s}$
\end{tabular}

\begin{tabular}{l|l}
\multicolumn{2}{l}{ Subscript } \\
\hline $\mathrm{C}$ & Compressor \\
con & control \\
$\mathrm{E}$ & Effect \\
$\mathrm{H}$ & Heat Chamber \\
$i$ & input \\
init & initialization \\
met & metal \\
$o$ & output \\
$\mathrm{P}$ & Pipes \\
$\mathrm{R}$ & Reservoirs and Plates \\
$\mathrm{T}$ & Tubes \\
$\mathrm{V}$ & vapor \\
$\mathrm{W}$ & water \\
$\Sigma$ & sum
\end{tabular}

for the mass flow and

$$
w_{o, \mathrm{~T}}(t)=\frac{\dot{m}_{i, \mathrm{~T}}\left(t-\tau_{\mathrm{T}}\right) w_{i, \mathrm{~T}}\left(t-\tau_{\mathrm{T}}\right)}{\dot{m}_{o, \mathrm{~T}}(t)}
$$

for the dry matter content. By assuming that the Compressor's thermal power equals its electrical power, or equivalently, the Compressor's efficiency factor is one, we obtain the relation

$$
\dot{m}_{\mathrm{v}}(t)=\bar{q} P_{\mathrm{C}}(t), \text { where } \bar{q}=\frac{\varrho_{\mathrm{v}, \mathrm{E}}}{p_{\mathrm{v}, \mathrm{H}}-p_{\mathrm{v}, \mathrm{E}}} .
$$

Of course, $\varrho_{\mathrm{v}, \mathrm{E}}, p_{\mathrm{v}, \mathrm{E}}$ and $p_{\mathrm{v}, \mathrm{H}}$ depend on $\vartheta_{\mathrm{E}}$ and $\vartheta_{\mathrm{H}}$, respectively. More precisely, the pressures $p_{\mathrm{v}, \mathrm{E}}, p_{\mathrm{v}, \mathrm{H}}$ are connected to the temperatures $\vartheta_{\mathrm{E}}, \vartheta_{\mathrm{H}}$ via the Antoine equation and the density $\varrho_{\mathrm{v}, \mathrm{E}}$ to the temperature $\vartheta_{\mathrm{E}}$ via the ideal gas law, cf. [24]. However, in practice, $\vartheta_{\mathrm{E}}$ is controlled to a fixed value and changes to $\vartheta_{\mathrm{H}}$ due to the Compressor's action are small, which is evident in [24]. Thus, assuming $\bar{q}=$ const is justified. Furthermore, since a slew rate limiter prevents fast changes of $P_{\mathrm{C}}$, the moving average filter term in (17) can be approximated by

$$
\frac{1}{\tau_{\mathrm{T}}} \int_{t-\tau_{\mathrm{T}}}^{t} \dot{m}_{\mathrm{v}}(\theta) \mathrm{d} \theta \approx \dot{m}_{\mathrm{v}}(t) \stackrel{(19)}{=} \bar{q} P_{\mathrm{C}}(t) .
$$

Note that (19) reveals that there exists a linear relation between the evaporating mass flow $\dot{m}_{\mathrm{v}}$ and the electrical power $P_{\mathrm{C}}$ supplied to the Compressor. As shown in [15], [24], the 
relation between $\dot{m}_{\mathrm{v}}$ and the Compressor's rotational speed $N_{\mathrm{C}}$ is nonlinear. From this simple consideration, it follows that it is more convenient to manipulate $P_{\mathrm{C}}$ instead of $N_{\mathrm{C}}$.

3) Reservoirs and Plates: By neglecting flash evaporation, which is small compared to other mass flows, and taking into account that FFEs essentially work around their operation point, we conclude that the dynamics of Reservoirs and Plates can be modeled via low-pass filters, see also [24], [25]. In this context, it is common to approximate a serial connection of low pass filters by a single low pass filter with an additional delay element. This additional delay is formally assigned to the Tubes and Pipes since their delays are dominant with regard to the whole process. Hence, the dynamics of Reservoirs and Plates are summed up by a low-pass filter w.r.t. mass flow such that

$$
\frac{\mathrm{d}}{\mathrm{d} t} \dot{m}_{o}(t)=\frac{1}{T_{2}}\left(\dot{m}_{i, \mathrm{R}}(t)-\dot{m}_{o}(t)\right)
$$

and a low pass filter w.r.t. dry matter content such that

$$
\frac{\mathrm{d}}{\mathrm{d} t} w_{o}(t)=\frac{1}{T_{1}}\left(w_{i, \mathrm{R}}(t)-w_{o}(t)\right) .
$$

4) Effect and Heat Chamber: In this section, we present simplified evaluations of the Effect's and Heat Chamber's energy balances. Compared to [24], the simplifications are as follows:

- The properties $c_{p}, \Delta h_{v}$, and $M$ of each liquid, water, and vapor are assumed constant and valid around the operation point.

- The Tubes of all four passes are bundled such that the sum of all tube surfaces $A_{\Sigma, \mathrm{T}}=\sum_{j=1}^{4} A_{\mathrm{T}, j}$, the average heat transfer coefficient $\bar{k}_{\mathrm{T}}=\sum_{j=1}^{4} k_{\mathrm{T}, j} / 4$ and the average specific heat capacity $\bar{c}_{p}=\left(c_{p, i}+c_{p, o}\right) / 2$ apply.

- The vapor mass flow $\dot{m}_{\mathrm{v} \text {,init }}$ to initialize the process is neglected since only operation around the stationary state is of interest.

- Heat losses to the ambience are neglected since they are small compared to all other energy flows.

- Boiling point elevation of the liquid is neglected for convenience.

Consequently, the energy balances yield

$$
\begin{aligned}
\frac{\mathrm{d}}{\mathrm{d} t} \vartheta_{\mathrm{E}}(t) & =\frac{\dot{q}_{i, \mathrm{E}}(t)+\dot{q}_{\mathrm{T}}(t)-\dot{q}_{o, \mathrm{E}}(t)-\dot{q}_{\mathrm{v}, \mathrm{C}}(t)}{M_{\mathrm{E}} \bar{c}_{p}+M_{\mathrm{met}, \mathrm{E}} c_{p, \text { met }}} \\
\frac{\mathrm{d}}{\mathrm{d} t} \vartheta_{\mathrm{H}}(t) & =\frac{\dot{q}_{\mathrm{v}, \mathrm{C}}(t)+P_{\mathrm{C}}(t)-\dot{q}_{\mathrm{T}}(t)-\dot{q}_{\mathrm{v}, \mathrm{con}}(t)-\dot{q}_{\mathrm{w}, \mathrm{H}}(t)}{M_{\mathrm{w}, \mathrm{H}} c_{p, \mathrm{w}, \mathrm{H}}+M_{\text {met }, \mathrm{H}} c_{p, \text { met }}},
\end{aligned}
$$

where

$$
\begin{aligned}
\dot{q}_{i, \mathrm{E}}(t) & =\dot{m}_{i}(t) c_{p, i} \vartheta_{\mathrm{E}}(t), \\
\dot{q}_{\mathrm{T}}(t) & =\bar{k}_{\mathrm{T}} A_{\Sigma, \mathrm{T}}\left(\vartheta_{\mathrm{H}}(t)-\vartheta_{\mathrm{E}}(t)\right), \\
\dot{q}_{o, \mathrm{E}}(t) & =\dot{m}_{o}(t) c_{p, o} \vartheta_{\mathrm{E}}(t), \\
\dot{q}_{\mathrm{v}, \mathrm{C}}(t) & =\bar{q} P_{\mathrm{C}}(t)\left(c_{p, \mathrm{w}, \mathrm{E}} \vartheta_{\mathrm{E}}(t)+\Delta h_{v, \mathrm{E}}\right), \\
\dot{q}_{\mathrm{v}, \mathrm{con}}(t) & =\dot{m}_{\mathrm{v}, \mathrm{con}}(t)\left(c_{p, \mathrm{w}, \mathrm{H}} \vartheta_{\mathrm{H}}(t)+\Delta h_{v, \mathrm{H}}\right), \\
\dot{q}_{\mathrm{w}, \mathrm{H}}(t) & =\left(\bar{q} P_{\mathrm{C}}(t)-\dot{m}_{\mathrm{v}, \mathrm{con}}(t)\right) c_{p, \mathrm{w}, \mathrm{H}} \vartheta_{\mathrm{H}}(t) .
\end{aligned}
$$

\section{B. Combination of Modules}

Based on Fig. 2, we combine (15)-(22) and thus obtain the input-output relations

$$
\begin{aligned}
\frac{\mathrm{d}}{\mathrm{d} t} w_{o}(t) & =\frac{1}{T_{1}}\left(\frac{w_{i}\left(t-\tau_{1}\right) \dot{m}_{i}\left(t-\tau_{2}\right)}{\dot{m}_{i}\left(t-\tau_{2}\right)-\bar{q} P_{\mathrm{C}}(t)}-w_{o}(t)\right), \\
\frac{\mathrm{d}}{\mathrm{d} t} \dot{m}_{o}(t) & =\frac{1}{T_{2}}\left(\dot{m}_{i}\left(t-\tau_{2}\right)-\bar{q} P_{\mathrm{C}}(t)-\dot{m}_{o}(t)\right),
\end{aligned}
$$

where $\tau_{1}=\tau_{\mathrm{T}}+\tau_{\mathrm{P}}$ and $\tau_{2}=\tau_{\mathrm{T}}$.

\section{Control Nomenclature and Nonlinear Model}

In this section, we introduce control nomenclature to distinguish between manipulated variables

$$
u(t)=\left[\begin{array}{c}
u_{1}(t) \\
u_{2}(t) \\
u_{3}(t)
\end{array}\right]:=\left[\begin{array}{c}
P_{\mathrm{C}}(t) \\
\dot{m}_{i}(t) \\
\dot{m}_{\mathrm{v}, \mathrm{con}}(t)
\end{array}\right],
$$

measured disturbances

$$
z(t):=w_{i}(t)
$$

states

$$
x(t)=\left[\begin{array}{l}
x_{1}(t) \\
x_{2}(t) \\
x_{3}(t) \\
x_{4}(t)
\end{array}\right]:=\left[\begin{array}{c}
w_{o}(t) \\
\dot{m}_{o}(t) \\
\vartheta_{\mathrm{E}}(t) \\
\vartheta_{\mathrm{H}}(t)
\end{array}\right]
$$

and controlled variables

$$
y(t)=\left[\begin{array}{l}
y_{1}(t) \\
y_{2}(t) \\
y_{3}(t)
\end{array}\right]:=\underbrace{\left[\begin{array}{llll}
1 & 0 & 0 & 0 \\
0 & 1 & 0 & 0 \\
0 & 0 & 1 & 0
\end{array}\right]}_{=C} x(t) .
$$

Hence, our model composed of (23) - (27) can be compactly denoted by

$$
\begin{aligned}
\frac{\mathrm{d}}{\mathrm{d} t} x(t) & =f\left(x(t), u(t), u\left(t-\tau_{2}\right), z\left(t-\tau_{1}\right)\right), \\
y(t) & =C x(t),
\end{aligned}
$$

which represents a nonlinear state space with input delays but linear output equation.

\section{Linearization}

To find a transfer function matrix representation, we linearize (32) about the operation point (OP) and get

$$
\begin{aligned}
\frac{\mathrm{d}}{\mathrm{d} t} \Delta x(t) & =\left.\frac{\partial f}{\partial x}\right|_{\mathrm{OP}} \Delta x(t)+\left.\frac{\partial f}{\partial u}\right|_{\mathrm{OP}} \Delta u(t) \\
& +\left.\frac{\partial f}{\partial u_{\tau_{2}}}\right|_{\mathrm{OP}} \Delta u\left(t-\tau_{2}\right)+\left.\frac{\partial f}{\partial z_{\tau_{1}}}\right|_{\mathrm{OP}} \Delta z\left(t-\tau_{1}\right) \\
\Delta y(t) & =C \Delta x(t)
\end{aligned}
$$

where $u_{\tau_{2}}:=u\left(t-\tau_{2}\right)$ and $z_{\tau_{1}}:=z\left(t-\tau_{1}\right)$ are abbreviations. The Laplace transformation of (33) neglecting initial values yields the transfer function matrix

$$
G(s)=C\left(s I_{4}-f_{x}\right)^{-1}\left(f_{u}+f_{u_{\tau_{2}}} \mathrm{e}^{-s \tau_{2}}\right)
$$


TABLE II: Parameters and operation point data [24]

\begin{tabular}{|c|c|c|}
\hline Symbol & Value & Unit \\
\hline$A_{\Sigma, \mathrm{T}}$ & 1139.7 & $\mathrm{~m}^{2}$ \\
\hline $\bar{c}_{p}$ & 3104 & $\mathrm{~J} \mathrm{~kg}^{-1} \mathrm{~K}^{-1}$ \\
\hline$c_{p, i}$ & 3280 & $\mathrm{~J} \mathrm{~kg}^{-1} \mathrm{~K}^{-1}$ \\
\hline$c_{p, \text { met }}$ & 477 & $\mathrm{~J} \mathrm{~kg}^{-1} \mathrm{~K}^{-1}$ \\
\hline$c_{p, o}$ & 2928 & $\mathrm{~J} \mathrm{~kg}^{-1} \mathrm{~K}^{-1}$ \\
\hline$c_{p, \mathrm{w}, \mathrm{E}}$ & 4184.8 & $\mathrm{~J} \mathrm{~kg}^{-1} \mathrm{~K}^{-1}$ \\
\hline$c_{p, \mathrm{w}, \mathrm{H}}$ & 4185.6 & $\mathrm{~J} \mathrm{~kg}^{-1} \mathrm{~K}^{-1}$ \\
\hline$\Delta h_{v, \mathrm{E}}$ & $2.396 \cdot 10^{6}$ & $\mathrm{~J} \mathrm{~kg}^{-1}$ \\
\hline$\Delta h_{v, \mathrm{H}}$ & $2.408 \cdot 10^{6}$ & $\mathrm{~J} \mathrm{~kg}^{-1}$ \\
\hline $\bar{k}_{\mathrm{T}}$ & 915 & $\mathrm{~W} \mathrm{~m}^{-2} \mathrm{~K}^{-1}$ \\
\hline$M_{\mathrm{E}}$ & 727.3 & $\mathrm{~kg}$ \\
\hline$M_{\text {met,E }}$ & 7000 & $\mathrm{~kg}$ \\
\hline$M_{\text {met, } \mathrm{H}}$ & 10000 & $\mathrm{~kg}$ \\
\hline$M_{\mathrm{w}, \mathrm{H}}$ & 1172 & $\mathrm{~kg}$ \\
\hline$p_{\mathrm{v}, \mathrm{E}}$ & 15.7 & $\mathrm{kPa}$ \\
\hline$p_{\mathrm{v}, \mathrm{H}}$ & 19.2 & $\mathrm{kPa}$ \\
\hline$T_{1}$ & 64 & $\mathrm{~s}$ \\
\hline$T_{2}$ & 101 & $\mathrm{~S}$ \\
\hline$\varrho_{\mathrm{v}, \mathrm{E}}$ & 0.1036 & $\mathrm{~kg} \mathrm{~m}^{-3}$ \\
\hline$\tau_{1}$ & 252 & $\mathrm{~s}$ \\
\hline$\tau_{2}$ & 200 & $\mathrm{~s}$ \\
\hline$\dot{m}_{i, \mathrm{OP}}$ & 6.6 & $\mathrm{~kg} \mathrm{~s}^{-1}$ \\
\hline$\dot{m}_{o, \mathrm{OP}}$ & 4.7521 & $\mathrm{~kg} \mathrm{~s}^{-1}$ \\
\hline$\dot{m}_{\mathrm{v}, \mathrm{con}, \mathrm{OP}}$ & 0.0121 & $\mathrm{~kg} \mathrm{~s}^{-1}$ \\
\hline$P_{\mathrm{C}, \mathrm{OP}}$ & 62250 & $\mathrm{~W}$ \\
\hline$w_{i, \mathrm{OP}}$ & 0.36 & $\mathrm{~kg} \mathrm{~kg}^{-1}$ \\
\hline$w_{o, \mathrm{OP}}$ & 0.5 & $\mathrm{~kg} \mathrm{~kg}^{-1}$ \\
\hline$\vartheta_{\mathrm{E}, \mathrm{OP}}$ & 55 & ${ }^{\circ} \mathrm{C}$ \\
\hline$\vartheta_{\mathrm{H}, \mathrm{OP}}$ & 59.25 & ${ }^{\circ} \mathrm{C}$ \\
\hline
\end{tabular}
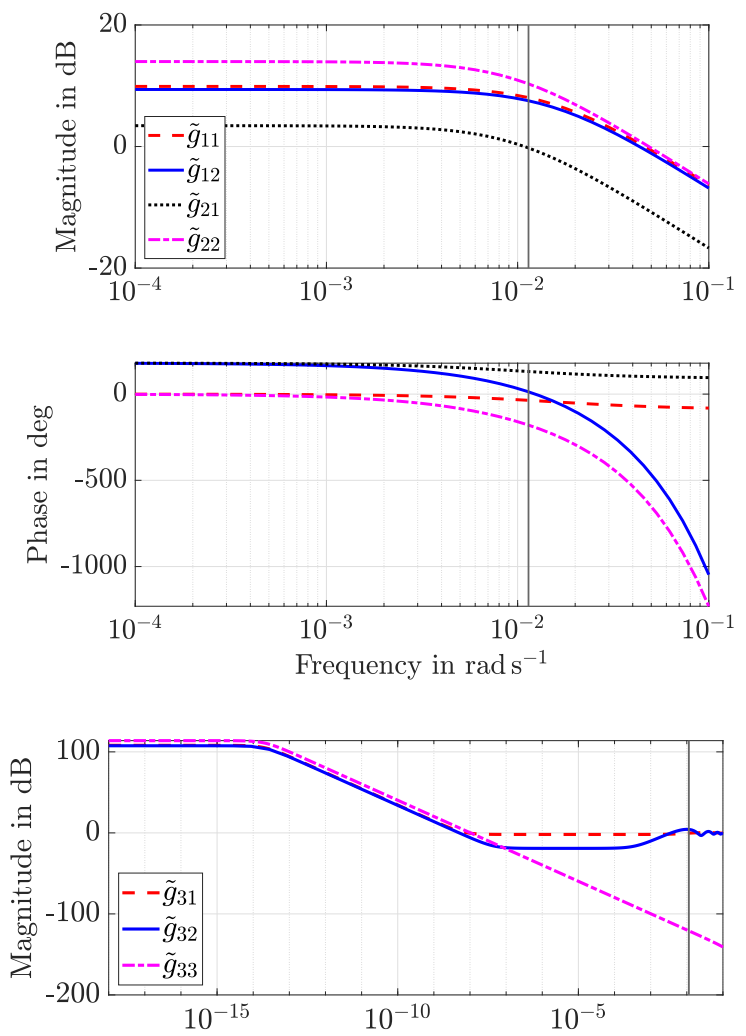

and the disturbance transfer function matrix

$$
G_{z}(s)=C\left(s I_{4}-f_{x}\right)^{-1} f_{z_{\tau_{1}}} \mathrm{e}^{-s \tau_{1}},
$$

where $f_{(\cdot)}:=\partial f /\left.\partial(\cdot)\right|_{\text {op. }}$ In the sequel, we focus on $G(s)$ appropriate (34) by applying the parameters and operation point data shown in TABLE II. The latter are either known from or identified via the validated full plant model in [24]. However, as parameter identification is not in the scope of this paper, we do not further detail this topic.

\section{LOOP PAIRING AND INTERACTION ANALYSES}

Besides some preliminaries, our main result is presented in this section, namely, the application of RGA, DRGA, NI, DIC, PM, and HIIA to the FFE process.

\section{A. Preliminaries}

Some of the analyses performed in this section depend on proper scaling of $G(s)$. To realize such scaling, there exist various opportunities [2]. Since FFEs essentially work in one operation point, it is useful to scale the outputs $y_{i}$ w.r.t. the largest allowed control error $e_{i \text {,max }}$, i.e.,

$$
\tilde{y}_{i}=\frac{y_{i}}{e_{i, \max }}, \quad i=1,2,3
$$

where $(\tilde{\cdot})$ indicates that $(\cdot)$ is scaled and

$$
\left[\begin{array}{l}
e_{1, \max } \\
e_{2, \max } \\
e_{3, \max }
\end{array}\right]=\left[\begin{array}{c}
0.01 \mathrm{~kg} \mathrm{~kg}^{-1} \\
0.2 \mathrm{~kg} \mathrm{~s}^{-1} \\
1^{\circ} \mathrm{C}
\end{array}\right] .
$$

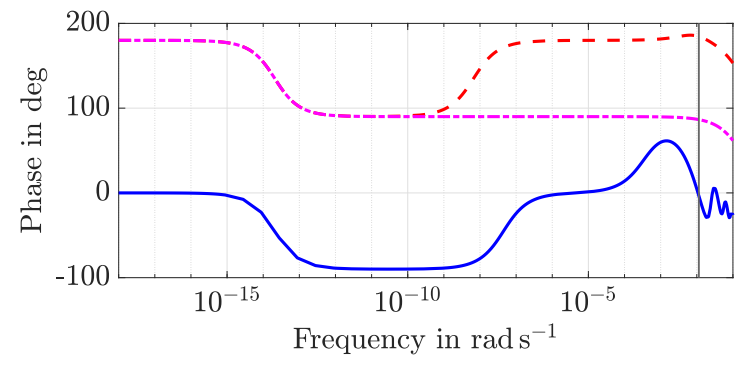

Fig. 3: Bode plots of the non-zero elements in $\tilde{G}(\mathrm{i} \omega)$

The inputs $u_{j}$ are scaled w.r.t. the largest allowed input change $u_{j, \max }$, i.e.,

$$
\tilde{u}_{j}=\frac{u_{j}}{u_{j, \max }}, \quad j=1,2,3
$$

with

$$
\left[\begin{array}{l}
u_{1, \max } \\
u_{2, \max } \\
u_{3, \max }
\end{array}\right]=\left[\begin{array}{c}
10 \mathrm{~kW}^{-1} \\
1 \mathrm{~kg} \mathrm{~s}^{-1} \\
0.1 \mathrm{~kg} \mathrm{~s}^{-1}
\end{array}\right] .
$$

To get an impression of the plant dynamics, we present the scaled transfer function matrix

$$
\tilde{G}(s)=\left[\begin{array}{lll}
\tilde{g}_{11}(s) & \tilde{g}_{12}(s) & \tilde{g}_{13}(s) \\
\tilde{g}_{21}(s) & \tilde{g}_{22}(s) & \tilde{g}_{23}(s) \\
\tilde{g}_{31}(s) & \tilde{g}_{32}(s) & \tilde{g}_{33}(s)
\end{array}\right]
$$


with

$$
\begin{aligned}
& \tilde{g}_{11}(s)=\frac{\tilde{\alpha}_{1}}{\tilde{\alpha}_{2}\left(\tilde{\alpha}_{3} s+1\right)}, \\
& \tilde{g}_{12}(s)=-\frac{\tilde{\alpha}_{4} \mathrm{e}^{-\tilde{\alpha}_{5} s}}{\tilde{\alpha}_{6}\left(\tilde{\alpha}_{3} s+1\right)}, \\
& \tilde{g}_{13}(s)=0, \\
& \tilde{g}_{21}(s)=-\frac{\tilde{\beta}_{1}}{\tilde{\beta}_{2}\left(\tilde{\beta}_{3} s+1\right)}, \\
& \tilde{g}_{22}(s)=\frac{\mathrm{e}^{-\tilde{\alpha}_{5} s}}{\tilde{\beta}_{3} s+1}, \\
& \tilde{g}_{23}(s)=0, \\
& \tilde{g}_{31}(s)=-\frac{\tilde{\gamma}_{1} s^{2}+\tilde{\gamma}_{2} s+\tilde{\gamma}_{3}}{\tilde{\gamma}_{4} s^{3}+\tilde{\gamma}_{5} s^{2}+\tilde{\gamma}_{6} s+\tilde{\gamma}_{7}}, \\
& \tilde{g}_{32}(s)=\frac{\tilde{\gamma}_{8} s^{2}+\tilde{\gamma}_{9} s+\tilde{\gamma}_{10}-\left(\tilde{\gamma}_{11} s+\tilde{\gamma}_{12}\right) \mathrm{e}^{-\tilde{\alpha}_{5} s}}{\tilde{\gamma}_{13} s^{3}+\tilde{\gamma}_{14} s^{2}+\tilde{\gamma}_{15} s+\tilde{\gamma}_{16}} \\
& \tilde{g}_{33}(s)=-\frac{\tilde{\gamma}_{17}}{\tilde{\gamma}_{18} s^{2}+\tilde{\gamma}_{19} s+\tilde{\gamma}_{20}}
\end{aligned}
$$

where $\tilde{\alpha}_{i}, \tilde{\beta}_{j}, \tilde{\gamma}_{k}$ denote positive constants. Analyzing (41a)(41i) shows that all denominator polynomials are Hurwitz. While the constants of the subsystem transfer functions $\tilde{g}_{i j}$ are not given for the sake of compact notation, we explicitly present the scaled steady-state gain matrix

$$
\tilde{G}(0)=\left[\begin{array}{ccc}
3.12 & -2.95 & 0 \\
-1.48 & 5 & 0 \\
-2.54 \cdot 10^{5} & 2.38 \cdot 10^{5} & -4.93 \cdot 10^{5}
\end{array}\right] .
$$

Let us briefly discuss the physical meaning of the negative elements in (42):

- $\tilde{g}_{12}(0)$ : Increasing $\dot{m}_{i}$, while keeping $P_{\mathrm{C}}$ constant, yields a larger denominator in (18) and thus decreases $w_{o}$.

- $\tilde{g}_{21}(0)$ : Increasing $P_{\mathrm{C}}$ leads to larger $\dot{m}_{\mathrm{v}}$, which, while keeping $\dot{m}_{i}$ constant, decreases $\dot{m}_{o}$, see (17).

- $\tilde{g}_{31}(0)$ : Increasing $P_{\mathrm{C}}$ initiates an energy flow from the Effect to the Heat Chamber, which, while keeping $\dot{m}_{i}$ and $\dot{m}_{\mathrm{v}, \text { con }}$ constant, decreases $\vartheta_{\mathrm{E}}$, see (23), (25d).

- $\tilde{g}_{33}(0)$ : Increasing $\dot{m}_{\mathrm{v}, \text { con }}$ initiates an energy flow out the Heat Chamber, see (24), (25e), which, while keeping $P_{\mathrm{C}}$ and $\dot{m}_{i}$ constant, decreases $\vartheta_{\mathrm{H}}$ and, via (25b), decreases $\vartheta_{\mathrm{E}}$, see (23).

The Bode plots of the non-zero elements in (40) are depicted in Fig. 3. Firstly, we observe that the inertia of $y_{3}$ is much larger than the one of $y_{1}$ and $y_{2}$. Secondly, according to (41h), $\tilde{g}_{32}$ is composed of a delayed part and a delay-free part. Since the delay-free part dominates the delayed part, the typical behavior of a delay element cannot be detected from the Bode plot of $\tilde{g}_{32}$. Thirdly, we can see that the plant crosses the phase angle of $180^{\circ}$ at $\omega_{\text {crit }}=0.0114 \mathrm{rad} \mathrm{s}^{-1}$ for the first time, see the black vertical line in Fig. 3. As mentioned in [15], $\omega_{\text {crit }}$ can be interpreted as the plant's crossover frequency and serves as rough orientation for the relevant operation domain, namely, at lower frequencies than $\omega_{\text {crit }}$.
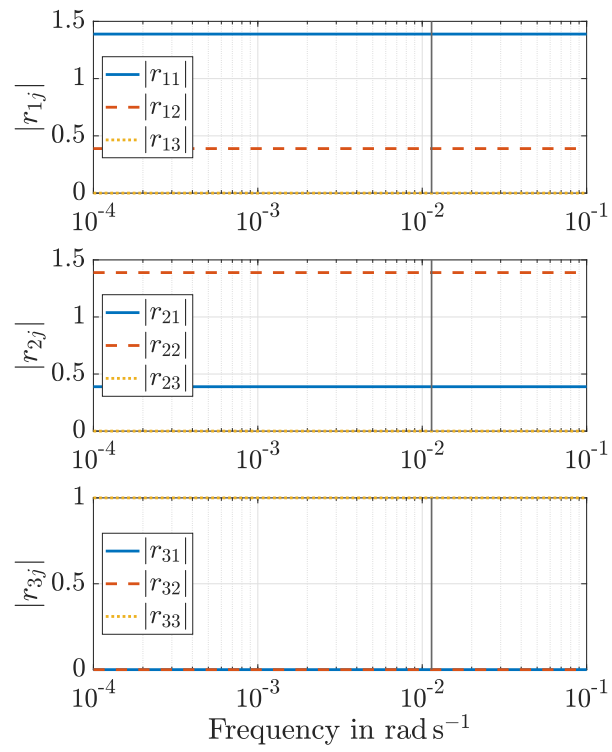

Fig. 4: Magnitudes of the dynamic relative gain array

\section{B. Results}

The steady-state RGA result is

$$
R(0)=\left[\begin{array}{ccc}
1.39 & -0.39 & 0 \\
-0.39 & 1.39 & 0 \\
0 & 0 & 1
\end{array}\right]
$$

and thus strongly recommends the diagonal pairing, i.e.,

$$
y_{1} \leftrightarrow u_{1}, \quad y_{2} \leftrightarrow u_{2}, \quad y_{3} \leftrightarrow u_{3} .
$$

By applying DRGA, we obtain the result in Fig. 4, where the black vertical line corresponds to $\omega_{\text {crit }}$, see Sec. IV-A. The DRGA shows a very good frequency stability and recommends the same pairing (44) as the steady-state RGA. A stand-alone interpretation of Fig. 4 would lead us to the wrong conclusion that the pairing $u_{2} \leftrightarrow y_{1}, u_{1} \leftrightarrow y_{2}, u_{3} \leftrightarrow y_{3}$ may also be feasible since $r_{12}$ and $r_{21}$ seem positive. However, from the steady-state RGA, we know that the latter are negative. Hence, it is important to always consider the DRGA in combination with the steady-state RGA result. Finally, by analytically calculating $R(s)$ with $\tilde{G}(s)$ according to (1), (40), we observe that $R(s)=R(0)=$ const for all $s$, which agrees with our numerical result in Fig. 4. Summing up, our steady-state RGA and DRGA analyses confirm the results in [15], where a similar-constructed FFE but a different model is investigated.

Evaluating the Niederlinski index of the (D)RGA paring (44) leads to

$$
\mathrm{NI}=0.72
$$

which is greater than zero and thus allows the conclusion that the pairing (44) may yield a stable multiloop SISO control system, see Sec. II-B1. 
From (43), it follows that the necessary condition (4) for DIC is fulfilled. The sufficient DIC condition (5) is also satisfied since

$$
\mu(E(0))=0.53
$$

which shows that $G(s)$ with pairing (44) is DIC. Hence, we have shown that multiloop SISO control with pairing (44) is feasible.

Fig. 3 underpins that there is strong interaction between $\tilde{g}_{11}$, $\tilde{g}_{12}, \tilde{g}_{21}$ and $\tilde{g}_{22}$, while (42), (43) indicate that controlling $y_{3}$ by $u_{3}$ is the only possibility. Therefore, we restrict the interaction analyses to the reduced plant

$$
\tilde{G}_{\text {red }}(s)=\left[\begin{array}{ll}
\tilde{g}_{11}(s) & \tilde{g}_{12}(s) \\
\tilde{g}_{21}(s) & \tilde{g}_{22}(s)
\end{array}\right] .
$$

Furthermore, in the following, delay terms in $\tilde{G}_{\text {red }}(s)$ are approximated via a fifth-order Padé approximation to enable application of the Gramian-based measures, cf. Sec. II-C.

Applying PM, see Sec. II-C1, and HIIA, see Sec. II-C2, to (47) leads to

$$
\bar{\Phi}=\left[\begin{array}{ll}
0.1383 & 0.3248 \\
0.0657 & 0.4712
\end{array}\right]
$$

and

$$
H=\left[\begin{array}{ll}
0.1728 & 0.2876 \\
0.0821 & 0.4574
\end{array}\right],
$$

respectively. The sum of the upper triangular in $\bar{\Phi}$ is 0.9343 and the sum of the upper triangular in $H$ is 0.9179 . Hence, both of these interaction measures recommend a sparse multivariable controller with upper triangular structure, i.e.,

$$
U(s)=\left[\begin{array}{cc}
K_{1}(s) & K_{3}(s) \\
0 & K_{2}(s)
\end{array}\right] E(s),
$$

where $E(s)$ is the Laplace transform of the control error vector. In this case, it seems beneficial to design $K_{3}(s)$ as decoupling feedforward. However, the control design is out of the present paper's scope.

\section{CONCLUSION AND OUTLOOK}

The results of this contribution are twofold. On the one hand, we have shown via RGA, DRGA, NI and DIC that multiple SISO loop control with the pairing (44) is feasible but, on the other hand, we deduce from PM and HIIA that the upper triangular multivariable controller (50) may be better to control the strongly interacting reduced plant (47). Hence, in the future, we intend to design such multivariable controller for the reduced plant, while the control of $y_{3}=\vartheta_{\mathrm{E}}$ is well realizable as SISO loop with $u_{3}=\dot{m}_{\mathrm{v}, \text { con }}$ as manipulated variable.

\section{REFERENCES}

[1] D. E. Seborg, T. F. Edgar, D. A. Mellichamp, and F. J. Doyle III, Process dynamics and control. John Wiley \& Sons, 2010.

[2] S. Skogestad and I. Postlethwaite, Multivariable feedback control: Analysis and design. John Wiley \& Sons, 2007.

[3] S. Engell, "Feedback control for optimal process operation," Journal of Process Control, vol. 17, no. 3, pp. 203-219, 2007.
[4] E. Bristol, "On a new measure of interaction for multivariable process control," IEEE Transactions on Automatic Control, vol. 11, no. 1, pp. 133-134, 1966.

[5] J. P. Gagnepain and D. E. Seborg, "Analysis of process interactions with applications to multiloop control system design," Industrial \& Engineering Chemistry Process Design and Development, vol. 21, no. 1, pp. 5-11, 1982.

[6] H. P. Huang, M. Ohshima, and I. Hashimoto, "Dynamic interaction and multiloop control system design," Journal of Process Control, vol. 4, no. 1, pp. 15-27, 1994.

[7] J. Oldenburg, H. J. Pallasch, C. Carroll, V. Hagenmeyer, S. Arora, K. Jacobsen, J. Birk, A. Polt, and P. van den Abeel, "Decision support for control structure selection during plant design," in Computer Aided Chemical Engineering. Elsevier, 2008, vol. 25, pp. 973-978.

[8] J. Hofmann, H. C. Holz, and L. Gröll, "Relative gain array and singular value analysis to improve the control in a biomass pyrolysis process," in 15th International Conference on Control and Automation. IEEE, 2019, pp. 596-603.

[9] F. Shinskey, "Predict distillation column response using relative gains," Hydrocarbon Processing, vol. 60, no. 5, pp. 196-200, 1981.

[10] A. Papadourakis, M. F. Doherty, and J. M. Douglas, "Relative gain array for units in plants with recycle," Industrial \& Engineering Chemistry Research, vol. 26, no. 6, pp. 1259-1262, 1987.

[11] J. Bao, P. J. McLellan, and J. F. Forbes, "A passivity-based analysis for decentralized integral controllability," Automatica, vol. 38, no. 2, pp 243-247, 2002

[12] A. Niederlinski, "A heuristic approach to the design of linear multivariable interacting control systems," Automatica, vol. 7, no. 6, pp. 691-701, 1971.

[13] A. Conley and M. E. Salgado, "Gramian based interaction measure," in 39th Conference on Decision and Control, vol. 5. IEEE, 2000, pp. 5020-5022.

[14] B. Wittenmark and M. E. Salgado, "Hankel-norm based interaction measure for input-output pairing," IFAC Proceedings Volumes, vol. 35, no. 1, pp. 429-434, 2002.

[15] J. Winchester and C. Marsh, "Dynamics and control of falling film evaporators with mechanical vapour recompression," Chemical Engineering Research and Design, vol. 77, no. 5, pp. 357-371, 1999.

[16] M. Hovd and S. Skogestad, "Controllability analysis for unstable processes," in Interactions Between Process Design and Process Control. Elsevier, 1992, pp. 49-54.

[17] S. Skogestad and M. Hovd, "Use of frequency-dependent RGA for control structure selection," in American Control Conference. IEEE, 1990, pp. 2133-2139.

[18] M. Hovd and S. Skogestad, "Simple frequency-dependent tools for control system analysis, structure selection and design," Automatica, vol. 28, no. 5, pp. 989-996, 1992.

[19] B. Halvarsson, "Interaction analysis in multivariable control systems: applications to bioreactors for nitrogen removal," Ph.D. dissertation, Acta Universitatis Upsaliensis, Sweden, 2010.

[20] P. Grosdidier, M. Morari, and B. R. Holt, "Closed-loop properties from steady-state gain information," Industrial \& Engineering Chemistry Fundamentals, vol. 24, no. 2, pp. 221-235, 1985.

[21] S. Skogestad and M. Morari, "Variable selection for decentralized control," Modeling, Identification and Control, vol. 13, no. 2, pp. 113$125,1992$.

[22] J. J. Belletrutti and A. G. J. MacFarlane, "Characteristic loci techniques in multivariable-control-system design," Proceedings of the Institution of Electrical Engineers, vol. 118, no. 9, pp. 1291-1297, 1971.

[23] J. Doyle, "Analysis of feedback systems with structured uncertainties," IEE Proceedings D (Control Theory and Applications), vol. 129, no. 6, pp. 242-250, 1982.

[24] C. Schwaer, J. Hofmann, M. Mühlpfordt, A. Frank, and L. Gröll, "Modular simulation model for falling film evaporators with novel approach to manage dominant time-varying transport delays," Computers \& Chemical Engineering, vol. 132, p. 106604, 2020.

[25] J. Hofmann, A. Ponomarev, V. Hagenmeyer, and L. Gröll, "Transport models for liquid films (in German)," at-Automatisierungstechnik, vol. 68, no. 8, pp. 625-640, 2020.

[26] J. Winchester, "Model based analysis of the operation and control of falling-film evaporators," Ph.D. dissertation, Massey University, New Zealand, 2000. 